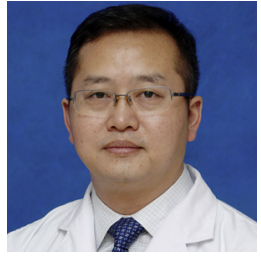

\section{VIDEO-ASSISTED RESECTION FOR LUNG CANCER RESULTS IN FEWER COMPLICATIONS To the Editor:}

By statistically analyzing the Society of Thoracic Surgeons General Thoracic Database, Boffa and colleagues demonstrate that video-assisted thoracic surgery (VATS) lung resection is associated with significantly fewer postoperative complications compared with thoracotomy in performing anatomic lung resection for curing non-small cell lung cancer $(P<.05) .{ }^{1}$ This conclusion reflects the evolution of VATS, including its birth, development, and maturity. This evolution can be traced back to the 1920s when Swedish physician Jacobaeus successfully dissected the intrathoracic adhesion by creating an artificial pneumothorax under cystoscopy for the treatment of chest tuberculosis. ${ }^{2}$ However, thoracoscopic surgery did not acquire a true vitality until the 1990s when the video-assisted thoracoscope was invented and the success of laparoscopic surgery had been widely accepted. From then on, the development of thoracoscopic surgery has moved to a fast track, gradually becoming a powerful tool and representative of the modern minimally invasive thoracic surgery. On the basis of the innovation of surgical instrumentation, surgeons' techniques have matured and VATS procedures have been optimized. The indications for VATS have been greatly extended, making it the most frequently applied method in thoracic surgery. Thoracotomy has gradually become the type of surgery for the "minority" of cases, although it still plays an irreplaceable role.

In 1992, Roviaro reported the first case of a complete endoscopic anatomic lobectomy, ${ }^{3}$ and in the following 20 years, experience and technique with the procedure developed. Today, the basic modes of VATS lung resection can be classified as "transfissure techniques" or "fissureless techniques." The first mode inherited the strategy and surgical approach from thoracotomy and is associated with a relatively higher incidence of postoperative air leak. An incomplete fissure will bring complications after VATS lung resection. Therefore, the fissureless approach aims to avoid dissecting the fissures and launches the dissection initially from the hilum. The single-direction VATS lobectomy, innovated by myself, is representative of this mode and has been widely adopted and applied throughout China. Thus, a successful exploration of the surgical mode can ensure the safety and efficacy of a VATS lobectomy. In their article, Boffa and colleagues emphasized that the statistical results were based on the Society of Thoracic Surgeons General Thoracic Database and reflected the outcome of the operations performed by boardcertified thoracic surgeons, which contributed to the lower incidence of morbidity and mortality compared with other databases (eg, the Healthcare Utilization Project and the Surveillance, Epidemiology, and End Results Medicare database), which included more heterogeneous surgeon backgrounds. These results, at least in a part, demonstrate the importance of developing an adequate capability for endoscopic surgical techniques and acquiring board certification.

Postoperative air leaks also were discussed, and it was a somewhat of a surprise that no significant differences were identified between the VATS cohort and the thoracotomy cohort. ${ }^{1}$ Except for dissecting fissure, the adhesions surrounding the bronchus and vessels at the hilum, and the enlarged lymph nodes at the interlobar space all will enforce the dissection into the nearby pulmonary parenchyma. As a result, air leaks will occur. Therefore, postoperative air leak is associated with the status of the fissure, the interlobar lymph nodes, and whether the dissected surface was well managed. As to whether the postoperative air leak had a clinical influence on the outcome with this mode of lung resection is another research subject.

Conversion can be ascribed to the complexity of the operation or to the unexpected intraoperative events, which most frequently is massive bleeding. Because of the growing abundance of VATS experiences, more complications during VATS have been overcome. Massive bleeding caused by vascular injury is considered the most troublesome and dangerous complication during VATS lung resection. We created a method called "suction-compressing angiorrhaphy technique," which has been proven to be helpful in controlling and managing intraoperative massive bleeding during endoscopy. The suction-compressing angiorrhaphy technique definitely helps avoid conversion when performing VATS lung resection. The VATS cohort remained less likely to experience a complication even when converted cases were excluded from the thoracotomy cohort.

This study represents the largest study to date to evaluate the treatment-associated morbidity in clinical stage I lung cancer. ${ }^{1}$ By applying the propensity-matched cohort study, the best statistical method in recent years for a retrospective analysis, this article demonstrates that the video-assisted approach to anatomic resection of clinical stage I lung cancer results in fewer complications. This is not only an essential and professional conclusion for this comparison after many years of VATS lung resection but also a consideration when counseling patients for surgery.

\section{Lunxu Liu, PhD, MD, FRCS Department of Thoracic Surgery West China Hospital Sichuan University Sichuan, People's Republic of China}

\section{References}

1. Boffa DJ, Dhamija A, Kosinski AS, Kim AW, Detterbeck FC, Mitchell JD, et al. Fewer complications result from a video-assisted approach to anatomic resection of clinical stage I lung cancer. J Thorac Cardiovasc Surg. 2014;148:637-43.

2. Jacobaeus HC. The cauterization of adhesions in artificial pneumothorax treat ment of pulmonary tuberculosis under thoracoscopic control. Pro R Soc Med 1923;16:45-62. 
3. Roviaro G, Rebuffat C, Varoli F, Vergani C, Mariani C, Maciocco M. Videoendoscopic pulmonary lobectomy for cancer. Surg Laparosc Endosc. 1992;2:244-7.

http://dx.doi.org/10.1016/j.jtcvs.2015.04.052

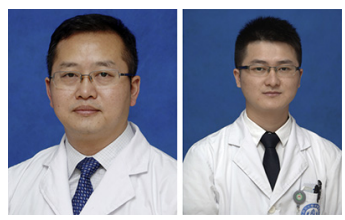

\section{NEED FOR CONVERSION OF VIDEO-ASSISTED THORACOSCOPIC SURGERY IS NOT AN INSURMOUNTABLE BARRIER}

To the Editor:

Video-assisted thoracoscopic surgery (VATS) is recommended as a surgical therapy for non-small cell lung cancer (NSCLC) that carries the advantages of minimal invasiveness and equivalent long-term efficacy to thoracotomy. ${ }^{1}$ It is difficult to learn the technology of VATS lobectomy, however, which limits its application in the world. The proportions of lobectomies performed by VATS are low both in China and in America, where VATS has been more developed. In their article in the Journal, Puri and colleagues ${ }^{2}$ have demonstrated that various causes, such as vascular issues, lymph nodes, anatomic considerations, and technical factors, may lead to conversions from VATS to thoracotomy. ${ }^{2}$ Possible need for conversion, however, should not be an insurmountable barrier. With the efforts of experts in the world, there are many effective methods to solve those complicated lobectomies, which used to cause conversions.

According to Puri and colleagues' analysis, ${ }^{2} 25 \%$ of conversions were related to vascular causes, 64\% related to anatomic considerations (such as pleural adhesions or large tumor), and $9 \%$ related to lymph nodes. ${ }^{2}$ With the accumulation of experience and maturation of skills, however, there are now well-established methods to handle these kinds of situations, most of which do not cause conversions nowadays. For most thoracic surgeons, partial pleural adhesions should have never been a hurdle impeding smooth VATS lobectomy. For cases with complete pleural adhesions, we can proceed with the procedure smoothly by constructing pleural tunnels between incisions with a finger. For cases with large tumors, it may be a little difficult to manipulate the lobe, and the space left for operating may be narrow. The method of "single-direction lobectomy" allows dissection of the lobe along the pulmonary hilum progressively while never flipping the lobe back and forth. ${ }^{3}$ This method makes resection of larger tumors more comfortable. With regard to situations with infiltration or adhesions of the tumor or lymph nodes, forced dissection should be avoided if possible to keep the vessels and bronchi from injury; thereafter, conversions can be avoided. Some experts have reported techniques of prophylactic clamping of the pulmonary artery. In case of potentially high risk of injury to adjacent structures as a result of discrete anatomy found during exploration, the pulmonary arterial trunk would be dissected and clamped, then dissection could be performed at leisure without any worry about catastrophic bleeding. Even if the vessels were injured, there would be no need to convert to thoracotomy. As a benefit of this technique, most complicated hila can be managed thoracoscopically. Vascular injuries are no longer an unexpected disaster. For cases with unexpected vascular injuries and massive hemorrhage, we developed the method of thoracoscopic "suction-compressing angiorrhaphy technique.", With this technique, we have successfully managed $88.24 \%$ of vascular injuries (15/17) thoracoscopically. ${ }^{4}$ The conversion rate for the 1040 cases of VATS lobectomy performed in our institution between 2006 and 2012 was only $1.73 \%$ $(18 / 1040)$ as a result of comprehensive use of the various effective methods mentioned here. ${ }^{5}$

Conversion is a problem that never hides when performing VATS lobectomy. As reported by Puri and colleagues ${ }^{2}$ and other experts, conversions from VATS to thoracotomy has never attenuated either perioperative nor long-term outcomes of patients. Conversion thus should never be a psychologic or technical hurdle for the surgeon attempting VATS lobectomy. Converting or not also depends on the capability of each surgeon, however, and on the surgeon's attitude toward tough situations. All thoracoscopic surgeons should go through a rigorous and systematic training program before they can truly benefit patients with this technique.

\section{Lunxu Liu, MD, PhD, FRCS Chengwu Liu, MD, PhD Department of Thoracic Surgery West China Hospital Sichuan University Chengdu, People's Republic of China}

\section{References}

1. Yan TD, Black D, Bannon PG, McCaughan BC. Systematic review and metaanalysis of randomized and nonrandomized trials on safety and efficacy of video-assisted thoracic surgery lobectomy for early-stage non-small-cell lung cancer. J Clin Oncol. 2009;27:2553-62.

2. Puri V, Patel A, Majumder K, Bell JM, Crabtree TD, Krupnick AS, et al. Intraoperative conversion from video-assisted thoracoscopic surgery lobectomy to open thoracotomy: a study of causes and implications. J Thorac Cardiovasc Surg. 2015;149:55-61. 62.e1.

3. Liu L, Che G, Pu Q, Ma L, Wu Y, Kan Q, et al. A new concept of endoscopic lung cancer resection: single-direction thoracoscopic lobectomy. Surg Oncol. 2010;19: e71-7

4. Mei J, Pu Q, Liao H, Ma L, Zhu Y, Liu L. A novel method for troubleshooting vascular injury during anatomic thoracoscopic pulmonary resection without conversion to thoracotomy. Surg Endosc. 2013;27:530-7.

5. Pu Q, Ma L, Che GW, Mei JD, Liao H, Wang Y, et al. [Safety and technical feasibility of single-direction VATS lobectomy: a review of 1040 cases.] Sichuan Da Хие Хие Bao Yi Xue Ban. 2013;44:109-13. Chinese.

http://dx.doi.org/10.1016/j.jtcvs.2015.06.003 